\title{
Rheological Properties of Roselle Seed Flour
}

\author{
Karma Bako Rimamcwe, U. D. Chavan* and P. M. Kotecha \\ Department of Food Science and Technology, Mahatma Phule Krishi Vidyapeeth, \\ Rahuri, India \\ *Corresponding author
}

\section{A B S T R A C T}

\section{Keywords}

Roselle seed flours, Sprouting, Decortication, Whole seed flour, Rheology, Amylograph, Farinograph and Extensograph

Article Info

Accepted:

17 June 2020 Available Online: 10 July 2020

\begin{abstract}
The present study was undertaken with the objectives to study the nutritional and functional qualities of Roselle seed flour and its utilization in bakery product by judging their rheological properties. For this study the Roselle seed were procured from the local market and then soaking, germination and decortications treatments were given to the seeds. All experiments were laid in completely randomized design with suitable replications for statistical analysis. The pre-treatments sprouting, decortications, and whole seed were adopted in this investigation to ascertain the best treatment for onward inclusion in bakery products. Amylograph, farinograph and extensograph properties of the pretreatments were also studied. The preliminary investigations adjudged decorticated Roselle seed flour (DRSF) have superior nutritional and functional qualities. Among the various treatments sprouted decorticated Roselle seed flour had higher nutrient contents than the whole seeds. The rheometry studies of Roselle seed oil (RSO) showed a shear thickening property, dilatancy a behavior rare in food materials. The economics of production of bakery products such as cookies with $15 \%$ Roselle seed flour and Roselle seed oil inclusion suggest that it is cost effective and affordable for the common man. Hence the need to commercialize Roselle seed flour extraction for industrial applications in food, medicine and allied products to maximize the value of Roselle seed.
\end{abstract}

\section{Introduction}

Roselle (Hibiscus sabdariffa Linn.) is a tropical plant belonging to the family Malvaceae and widely cultivated for its jute like fiber in India, the East Indies, Nigeria and to some extent in tropical America (Yayock, 1988). A woody sub-shrub growing 7-8 feet $(2-2.5 \mathrm{~m})$ tall, acting as annual or perennial, takes about six months to mature. The mature plants are highly drought resistant but may require water during dry periods when soil moisture is depleted to the point where wilting occurs. Roselle requires a chalky, loamy and peat-rich soil with $\mathrm{pH}$ of 7.6-9.0; and grows best in weakly alkaline soil (Myfolia, 2016)

The seeds of this crops from which edible oil could be extracted are being wasted in its production area after the farmers might have taken the quantity needed for the next planting season for the calyces production (Bamigboy et al., 2009; Emmy Hainida et al., 
2008). The seeds have been found to be a source of highly valued vegetable oil with properties similar to that of crude olive. In terms of their oil content, the seeds of $H$. sabdariffa are richer in lipids (22\%) than most well-known seed oil such as those derived from Cotton (13\%), Soybean (14\%) and Palm fruit (20\%) (Nzikou et al., 2012).

Nutritionally important antioxidants such as tocopherols improve the stability of oil. In a study, Roselle seed oil (RSO) and Roselle seed extract (RSE) was mixed with Sunflower oil, respectively to monitor degradation rate and investigate antioxidant activity during accelerated storage. The antioxidant activity was found to stabilize Sunflower oil of various samples and in the order of RSE > RSO > Tocopherol > Sunflower oil (Nyam et al., 2012).

Roselle seed oil are richer in carotenoids than expensive oils like niger (Guizotia abyssinica) seed oil $(70.2 \pm 0.03 \mathrm{mg} \beta$-carotene $/ 100 \mathrm{~g})$ and coriander (Coriander sativum) seed oil (89.2 $\pm 0.05 \mathrm{mg} \quad \beta$-carotene/100g) (Ramadan and Morsel, 2004). Carotenoids are important ingredients in cosmetic industries due to their antioxidant activity and protective effect on the skin (Platon, 1997). Therefore, Roselle seeds oil has good potential for utilization in the Cosmetic Industry. The proximate composition of whole Roselle seeds indicated that, seeds contained relatively high fat and protein (as \%N $\times 6.25 ; 20.97 \%$ and $29.61 \%$ respectively). The physico-chemical parameters of crude oil extracted from Roselle seeds by soaking at room temperature (cold extraction) indicated the oils had 1.4674 refractive index; 0.078 (at 420nm) yellowgreenish colour, $0.78 \%$ acidity, 198.82 saponification value, 97.62 ( $\mathrm{g}$ of $\mathrm{I}_{2} / 100 \mathrm{~g}$ oil) iodine value; $1.52 \%$ unsaponifiable matter; 4.82 (Meq $0_{2} / \mathrm{Kg}$ oil) peroxide value; $6.21 \mathrm{p}$ anisidine value; and 15.85 totox number. Gas Liquid Chromatography technique has been developed for identification and quantitative determination of total unsaturated and saturated fatty acids. This technology showed that Roselle crude oil had $73.40 \%$ unsaturated and $26.57 \%$ saturated fatty acids respectively. Major fatty acid found was oleic acid (38.46\%) followed by linoleic $(33.25 \%)$ and Stearic $(5.79 \%)$. Stability of crude Roselle seed oil against oxidation during the accelerated storage of oil indicated that the crude oil induction period to be 10 days at $65^{\circ} \mathrm{C}$. The relatively high fat content of the seeds and high protein content of resulted meal beside the relatively high oxidation stability of Roselle suggest that Roselle seeds could be a novel and economic source of healthy edible fat and for other food industry applications.

Protein fractions, proteins isolates or concentrates obtained from Roselle seeds might be an alternative source of low cost protein substitute in dietary supplement in ingredients for food industry. This may reduce the heavy dependence on conventional sources such as animal, fish and soybean proteins. At present, there are very few reports on harnessing the bio-nutritional potential of Roselle seeds in value added products (Nyam et al., 2014). Adding cereals with complementary nutritive profiles, such as Roselle seeds, may yield a more complete enrich food source (Bala et al., 2015; Wani et al., 2015, Bako Karma et al., 2019). Hence, the aim of this study was to investigate the Rheological properties of Roselle seed flour for utilization in various food products.

\section{Materials and Methods}

Roselle (Hibiscus sabdariffa Linn.) and Wheat (Triticum aestivum spp.) seeds were both sourced from local vegetable markets at Ahmednagar, Maharashtra State, India and both were of local varieties. 


\section{Cleaning}

The seeds were cleaned by washed to separate poor quality seeds, adhering dust particles, stones, plant debris and were dried carefully at ambient conditions to preserve its nutritive value, packed in a HDPE bag and stored in a cool dry place until further used.

\section{Germination}

The cleaned seeds were soaked for 6 hours to activate the process of germination, after which the seeds were washed and allowed to drain. The drained seeds were then spread on a damped cloth in a perforated container with water sprinkled occasionally in a dark room to activate germination for a another 12 hour period; then gently washed and spread sparsely to dry under fan at ambient temperature to preserve its nutritive value.

\section{Decortication}

The seed sample was soaked in distilled water for a period of $6 \mathrm{hrs}$ and drained. The seed was pressed between hand fingers to neatly separate out the bran (seed coat) from the cotyledons. The bran and cotyledons were dried at $105^{\circ} \mathrm{C}$ for $3 \mathrm{hrs}$ in an oven. The dry weight was then obtained using Sartorius AG Germany (Model: CPA323S-0CE) digital weighing balance of $0.001 \mathrm{~g}$ accuracy, and the percentage was calculated and recorded. All above samples packed in a HDPE bag and stored in a cool dry place until further use.

To ascertain the best treatment of Roselle seeds to be selected for composite Roselle:Wheat flour formulation in cookie production for an enhanced nutritional and functional properties of the value added product, the following preliminary studies on pre-treatments where adopted for Roselle seeds.
Whole Roselle Seeds Flour (WRSF), as Control

Decorticated Roselle Seed flour (UDRSF)

Sprouted Whole Roselle Seed Flour (SWRSF)

Sprouted Decorticated Roselle Seed Flour (SDRSF)

These pre-treatments were prepared accordingly: both cleaned raw and germinated (sprouted) seeds were divided into two portion each, the first portion was grind whole with a laboratory scale hammer mill and the resulting powder sieved through a 60 mesh screen filter until a fine whole seed powder was obtained; the second portion was used to separate out the bran to obtain a decorticated flour then further grind to a fine flour. The resulting whole and decorticated cleaned (raw and sprouted) seed flour fractions obtained were packed separately in a HDPE bag and stored in a cool dry place until used.

\section{Results and Discussion}

\section{Rheological studies}

An in-depth literature search revealed that the rheological effects of incorporating Roselle seed flour in a dough composite formulation with wheat flour have not yet been studied. Thus this study was conducted to investigate the rheological behaviour of the composite flour to ascertain the baking properties and potentials of the flour mixtures. The results for the Amylograph, Farinograph and Extensograph are outlined below:

\section{Amylograph studies}

The gelatinization and pasting characteristics of the composite flours (Roselle seed flour and wheat flour) were studied. The results outlined showed that the baking properties of 
flour are based not only on the gluten characteristics but also to a great degree on the extent of gelatinization of the starch. The porosity and the crumb structure of the baked goods depend upon this factor which also determines the degree of firmness and the shelf life of the product (AACC, 2000 standard method 22-10).

The results of amylograph of composite flour (Roselle seed flour and wheat flour) are presented in Table 1-4 and figures 1-5 for the characteristics: beginning of gelatinization, gelatinization temperature and gelatinization maximum. The results showed that the higher percentage of Roselle seed flour (RSF) inclusion in the composite mix the greater increased temperature required beginning gelatinization of the composite slurry in all the treatments, with a corresponding decrease in gelatinization temperature showing an inverse relationship. The difference in gelatinization temperatures between each of the treatment were considered significant at $\mathrm{P}<0.05$; but $10 \%$ and $15 \%$ substitution were at par with control.

The difference in beginning of gelatinization and gelatinization temperature of the slurry decreases with inclusion of RSF which reflects the degree and the extent of gelatinization. Ten (10\%) percept Roselle seed inclusion showed a higher deviation followed by $15 \%$ and with $25 \%$ having the least value. There was a significant difference in the temperature $(\mathrm{P}>0.05)$ between each treatments. This could be as a result of reduction in overall starch and gluten contents of the composite flour which is responsible for the gelling and viscoelastic properties to the flour. Roselle seed has high protein content and as such inclusion in the composite mix will only enrich the nutrient value of the flour. Gelatinization Maximum [BU] also decreased in all treatments with the inclusion of RSF; with $25 \%$ inclusion having the least values. However, in all the treatments the amylogram reveals that the samples meets the standard quality of bread rye, because all the gelatinization temperature was above $63^{\circ} \mathrm{C}$ and the gelatinization maximum was above 200 AU (AACC, 2000 Standard Method No. 22-10).

\section{Farinograph studies}

For decades the farinograph has been the standard instrument for measuring the water absorption, development time, stability, mixing tolerance index (MTI), time to break down and the farinograph quality number of the flour and whole meal, as well as testing the mixing and processing behavior of dough. These values extracted from the farinograph study can be used as a relative composite description of flour's overall quality characteristics. This has been used worldwide in accordance to international standard (AACC, 2000 Method No. 54-21). Farinograph results for composite flour of Roselle seed flour and wheat flour treatments are shown in Table 5-8. Based on the descriptive categories outlined by Preston (1984), the results obtained from farinograph studies outlined in Tables 5-8 of the composite flour formulations can be categorized as follows:

For whole Roselle seed flour (WRSF), farinograph studies revealed that the range $10 \%, 15 \%, 20 \%$ and $25 \%$ composite flour can be categorized as strong and medium flours respectively (Preston, 1984), with 10\% and $15 \%$ compared favourably with the control as strong flours. Having MTI values of 46.0, 52.5 and $31.75 \mathrm{BU}$; development time 6.0, 5.9 and $6.78 \mathrm{~min}$; and water absorption 78.63, 76.60 and $79.58 \%$ respectively (Table 5); there was a significant difference $(\mathrm{P}<0.05)$ between the observed values of dough characteristic of all the treatment formulations. Water absorption, development 
time, stability, and farinograph quality number (FQN) values decreased with inclusion of WRSF in the composite mixture, with the exception of MTI [BU] showing an inverse relationship.

For decorticated Roselle seed flour (DRSF), farinogram classified the composite flour with $10 \%, 15 \%, 20 \%$ and $25 \%$ as strong, medium and weak flours respectively (Preston, 1984), with only $10 \%$ compared favourably with control as strong flours; having MTI values of
54.25 and $31.75 \mathrm{BU}$, development time 5.6 and $6.78 \mathrm{~min}$, and water absorption 76.33 and $79.58 \%$ respectively (Table 6). There is a significant difference $(\mathrm{P}<0.05)$ between the observed values of dough characteristic of all the treatment formulations. Water absorption, development time, stability and farinograph quality number (FQN) values decreased with the inclusion of DRSF in the composite mixture, with the exception of MTI [BU] showing an inverse relationship.

Table.1 Effects of addition of whole Roselle seeds flour (WRSF) on amylograph characteristic of dough

\begin{tabular}{|c|c|c|c|}
\hline $\begin{array}{l}\text { Treatments } \\
\text { Roselle seed flour:wheat }\end{array}$ & $\begin{array}{c}\text { Beginning of } \\
\text { gelatinization }\left({ }^{0} \mathrm{C}\right)\end{array}$ & $\begin{array}{l}\text { Gelatinization } \\
\text { temperature }\left({ }^{0} \mathrm{C}\right)\end{array}$ & $\begin{array}{l}\text { Gelatinization } \\
\text { maximum (AU) }\end{array}$ \\
\hline Control (00:100) & 59.58 & 87.08 & 838.75 \\
\hline WRSF 10:90 & 60.73 & 84.53 & 563.25 \\
\hline WRSF 15:85 & 61.88 & 84.48 & 467.75 \\
\hline WRSF 20:80 & 62.33 & 83.95 & 413.50 \\
\hline WRSF 25:75 & 63.48 & 83.65 & 348.00 \\
\hline $\operatorname{SE}( \pm)$ & 0.04 & 0.05 & 0.47 \\
\hline CD at5 (\%) & 0.14 & 0.15 & 1.42 \\
\hline CV (\%) & 0.16 & 0.12 & 0.18 \\
\hline
\end{tabular}

Each value is an average of four determinations. WRSF $=$ Whole Roselle Seed Flour

Table.2 Effects of addition of decorticated Roselle seeds flour (DRSF) on amylograph characteristic of dough

\begin{tabular}{|c|c|c|c|}
\hline $\begin{array}{l}\text { Treatments } \\
\text { Roselle seed flour:wheat }\end{array}$ & $\begin{array}{c}\text { Beginning of } \\
\text { gelatinization }\left({ }^{0} \mathrm{C}\right)\end{array}$ & $\begin{array}{c}\text { Gelatinization } \\
\text { temperature }\left({ }^{0} \mathrm{C}\right)\end{array}$ & $\begin{array}{c}\text { Gelatinization } \\
\text { maximum (AU) }\end{array}$ \\
\hline Control (00:100) & 59.58 & 87.08 & 838.75 \\
\hline DRSF 10:90 & 61.05 & 86.23 & 605.25 \\
\hline DRSF 15:85 & 62.38 & 85.38 & 525.75 \\
\hline DRSF 20:80 & 62.55 & 84.93 & 466.75 \\
\hline DRSF 25:75 & 64.08 & 84.88 & 392.75 \\
\hline $\mathrm{SE}( \pm)$ & 0.04 & 0.05 & 0.48 \\
\hline CD at5 (\%) & 0.15 & 0.14 & 1.44 \\
\hline CV $(\%)$ & 0.16 & 0.11 & 0.17 \\
\hline
\end{tabular}

Each value is an average of four determinations. DRSF $=$ Decorticated Roselle Seed Flour 
Table.3 Effects of addition of sprouted whole Roselle seeds flour (SWRSF) on amylograph characteristic of dough

\begin{tabular}{|c|c|c|c|}
\hline $\begin{array}{l}\text { Treatments } \\
\text { Roselle seed flour:wheat }\end{array}$ & $\begin{array}{c}\text { Beginning of } \\
\text { gelatinization }\left({ }^{0} \mathrm{C}\right)\end{array}$ & $\begin{array}{c}\text { Gelatinization } \\
\text { temperature }\left({ }^{0} \mathrm{C}\right)\end{array}$ & $\begin{array}{l}\text { Gelatinization } \\
\text { maximum }(A U)\end{array}$ \\
\hline Control (00:100) & 59.58 & 87.08 & 838.75 \\
\hline SWRSF 10:90 & 61.03 & 85.55 & 648.25 \\
\hline SWRSF 15:85 & 61.48 & 84.58 & 504.50 \\
\hline SWRSF 20:80 & 62.83 & 84.23 & 488.75 \\
\hline SWRSF 25:75 & 63.93 & 84.15 & 403.50 \\
\hline $\mathrm{SE}( \pm)$ & 0.36 & 0.07 & 0.52 \\
\hline CD at5 (\%) & 1.09 & 0.20 & 1.57 \\
\hline CV $(\%)$ & 1.17 & 0.16 & 0.18 \\
\hline
\end{tabular}

Each value is an average of four determinations. SWRSF $=$ Sprouted Whole Roselle Seed Flour

Table.4 Effects of addition of sprouted decorticated Roselle seeds flour (SDRSF) on amylograph characteristic of dough

\begin{tabular}{|c|c|c|c|}
\hline $\begin{array}{l}\text { Treatments } \\
\text { Roselle seed flour:wheat }\end{array}$ & $\begin{array}{c}\text { Beginning of } \\
\text { gelatinization }\left({ }^{0} \mathrm{C}\right)\end{array}$ & $\begin{array}{c}\text { Gelatinization } \\
\text { temperature }\left({ }^{0} \mathrm{C}\right)\end{array}$ & $\begin{array}{r}\text { Gelatinization } \\
\text { maximum (AU) }\end{array}$ \\
\hline Control (00:100) & 59.58 & 87.08 & 838.75 \\
\hline SDRSF 10:90 & 61.33 & 86.00 & 624.25 \\
\hline SDRSF 15:85 & 61.88 & 85.38 & 576.50 \\
\hline SDRSF 20:80 & 63.03 & 85.03 & 484.25 \\
\hline SDRSF 25:75 & 63.68 & 84.70 & 404.75 \\
\hline $\mathrm{SE}( \pm)$ & 0.05 & 0.13 & 0.61 \\
\hline CD at5 (\%) & 0.14 & 0.40 & 1.83 \\
\hline CV (\%) & 0.15 & 0.31 & 0.21 \\
\hline
\end{tabular}

Each value is an average of four determinations. SDRSF $=$ Sprouted Decorticated Roselle Seed Flour 
Table.5 Effects of addition of whole Roselle seeds flour (WRSF) on farinograph characteristics of dough

\begin{tabular}{|c|c|c|c|c|c|c|c|c|c|}
\hline $\begin{array}{l}\text { Treatments } \\
\text { Roselle seed } \\
\text { flour:wheat }\end{array}$ & Consistency [BU] & $\begin{array}{c}\text { Water } \\
\text { absorption \% }\end{array}$ & $\begin{array}{c}\text { Water } \\
\text { absorption } \\
\text { (corrected for } \\
\mathbf{5 0 0} \mathbf{~ B U )}\end{array}$ & $\begin{array}{c}\text { Water } \\
\text { absorption } \\
\text { (corrected for } \\
14 \%)\end{array}$ & $\begin{array}{l}\text { Dev. } \\
\text { time } \\
(\text { min) }\end{array}$ & $\begin{array}{l}\text { Stability } \\
\text { (min) }\end{array}$ & $\begin{array}{c}\text { Mixing } \\
\text { Tolerance } \\
\text { Index (BU) }\end{array}$ & $\begin{array}{l}\text { Time of } \\
\text { break } \\
\text { down } \\
\text { (min) }\end{array}$ & $\begin{array}{l}\text { Farinograph } \\
\text { quality number } \\
\text { (FQN) }\end{array}$ \\
\hline Control (00:100) & 498.75 & 85.18 & 85.08 & 79.58 & 6.78 & 3.98 & 31.75 & 11.68 & 116.75 \\
\hline WRSF 10:90 & 494.00 & 83.65 & 83.45 & 78.63 & 6.00 & 2.88 & 46.00 & 8.60 & 85.50 \\
\hline WRSF 15:85 & 505.50 & 81.03 & 81.20 & 76.60 & 5.90 & 2.33 & 52.50 & 8.28 & 82.75 \\
\hline WRSF 20:80 & 503.25 & 79.55 & 79.63 & 74.55 & 5.65 & 1.95 & 63.95 & 7.35 & 74.00 \\
\hline WRSF 25:75 & 502.50 & 78.48 & 78.50 & 73.38 & 5.50 & 1.68 & 89.50 & 7.08 & 70.25 \\
\hline $\mathbf{S E}( \pm)$ & 0.96 & 0.04 & 0.04 & 0.04 & 0.04 & 0.04 & 0.34 & 0.04 & 0.39 \\
\hline CD at5 (\%) & 2.90 & 0.11 & 0.13 & 0.13 & 0.12 & 0.12 & 1.01 & 0.13 & 1.18 \\
\hline CV $(\%)$ & 0.38 & 0.09 & 0.10 & 0.11 & 1.35 & 3.19 & 1.18 & 1.01 & 0.91 \\
\hline
\end{tabular}

Each value is an average of four determinations. WRSF $=$ Whole Roselle Seed Flour

Table.6 Effects of Addition of decorticated Roselle seeds flour (DRSF) on farinograph characteristics of dough

\begin{tabular}{|c|c|c|c|c|c|c|c|c|c|}
\hline $\begin{array}{l}\text { Treatments } \\
\text { Roselle seed } \\
\text { flour:wheat }\end{array}$ & $\begin{array}{c}\text { Consistency } \\
\text { [BU] }\end{array}$ & $\begin{array}{c}\text { Water } \\
\text { absorpti } \\
\text { on \% }\end{array}$ & $\begin{array}{c}\text { Water } \\
\text { absorption } \\
\text { (corrected } \\
\text { for } 500 \mathrm{BU} \text { ) }\end{array}$ & $\begin{array}{c}\text { Water } \\
\text { absorption } \\
\text { (corrected } \\
\text { for } 14 \% \text { ) }\end{array}$ & $\begin{array}{l}\text { Dev. } \\
\text { time } \\
\text { (min) }\end{array}$ & $\begin{array}{l}\text { Stability } \\
\text { (min) }\end{array}$ & $\begin{array}{l}\text { Mixing } \\
\text { Tolerance } \\
\text { Index } \\
\text { (BU) }\end{array}$ & $\begin{array}{c}\text { Time of } \\
\text { break } \\
\text { down } \\
\text { (min) }\end{array}$ & $\begin{array}{c}\text { Farinograph } \\
\text { quality } \\
\text { number (FQN) }\end{array}$ \\
\hline Control (00:100) & 498.75 & 85.18 & 85.08 & 79.58 & 6.78 & 3.98 & 31.75 & 11.68 & 116.75 \\
\hline DRSF 10:90 & 489.25 & 82.13 & 81.85 & 76.33 & 5.60 & 2.43 & 54.25 & 7.53 & 75.50 \\
\hline DRSF 15:85 & 511.00 & 79.98 & 80.38 & 75.05 & 5.28 & 2.28 & 66.75 & 7.08 & 70.75 \\
\hline DRSF 20:80 & 506.00 & 78.80 & 79.03 & 73.73 & 4.93 & 1.58 & 85.25 & 6.35 & 63.25 \\
\hline DRSF 25:75 & 7.70 & 78.38 & 78.58 & 73.00 & 4.80 & 1.23 & 125.50 & 5.83 & 58.50 \\
\hline SE $( \pm)$ & 1.21 & 0.05 & 0.04 & 0.05 & 0.49 & 0.04 & 0.48 & 0.05 & 0.41 \\
\hline CD at5 (\%) & 3.64 & 0.15 & 0.14 & 0.14 & 0.16 & 0.13 & 1.46 & 0.12 & 1.25 \\
\hline CV $(\%)$ & 0.60 & 0.12 & 0.12 & 0.12 & 1.90 & 3.86 & 1.33 & 1.06 & 1.07 \\
\hline
\end{tabular}

Each value is an average of four determinations. DRSF $=$ Decorticated Roselle Seed Flour 
Table.7 Effects of addition of sprouted whole Roselle seeds flour (SWRSF) on farinograph characteristics of dough

\begin{tabular}{|c|c|c|c|c|c|c|c|c|c|}
\hline $\begin{array}{l}\text { Treatments } \\
\text { Roselle seed } \\
\text { flour:wheat }\end{array}$ & $\begin{array}{l}\text { Consiste } \\
\text { ncy [BU] }\end{array}$ & $\begin{array}{c}\text { Water } \\
\text { absorption } \\
\%\end{array}$ & $\begin{array}{c}\text { Water } \\
\text { absorption } \\
\text { (corrected } \\
\text { for } 500 \text { BU) }\end{array}$ & $\begin{array}{c}\text { Water } \\
\text { absorption } \\
\text { (corrected } \\
\text { for } 14 \% \text { ) }\end{array}$ & $\begin{array}{l}\text { Dev. } \\
\text { time } \\
(\min )\end{array}$ & $\begin{array}{l}\text { Stability } \\
\text { (min) }\end{array}$ & $\begin{array}{l}\text { Mixing } \\
\text { Tolerance } \\
\text { Index } \\
\text { (BU) }\end{array}$ & $\begin{array}{l}\text { Time of } \\
\text { break } \\
\text { down } \\
\text { (min) }\end{array}$ & $\begin{array}{c}\text { Farinograph } \\
\text { quality number } \\
\text { (FQN) }\end{array}$ \\
\hline Control (00:100) & 498.75 & 85.18 & 85.08 & 79.58 & 6.78 & 3.98 & 31.75 & 11.68 & 116.75 \\
\hline SWRSF 10:90 & 491.00 & 81.13 & 80.93 & 76.73 & 5.60 & 2.60 & 42.00 & 9.03 & 91.75 \\
\hline SWRSF 15:85 & 507.00 & 78.98 & 79.18 & 74.93 & 6.08 & 2.48 & 44.25 & 8.38 & 83.75 \\
\hline SWRSF20:80 & 511.00 & 77.13 & 77.43 & 72.95 & 5.65 & 2.30 & 45.00 & 7.98 & 79.25 \\
\hline SWRSF25:75 & 504.75 & 76.50 & 76.68 & 71.48 & 5.38 & 1.70 & 65.25 & 7.70 & 77.50 \\
\hline SE $( \pm)$ & 0.70 & 0.04 & 0.04 & 0.05 & 0.05 & 0.06 & 0.34 & 0.05 & 0.52 \\
\hline CD at5 (\%) & 2.11 & 0.14 & 0.14 & 0.16 & 0.15 & 0.16 & 1.04 & 0.13 & 1.56 \\
\hline CV $(\%)$ & 0.28 & 0.12 & 0.12 & 0.14 & 1.17 & 4.14 & 1.51 & 0.96 & 1.15 \\
\hline
\end{tabular}

Each value is an average of four determinations. SWRSF $=$ Sprouted Whole Roselle Seed Flour

Table.8 Effects of addition of sprouted decorticated Roselle seeds flour (SDRSF) on farinograph characteristics of dough

\begin{tabular}{|c|c|c|c|c|c|c|c|c|c|}
\hline $\begin{array}{l}\text { Treatments } \\
\text { Roselle seed flour:wheat }\end{array}$ & $\begin{array}{c}\text { Consist } \\
\text { ency } \\
\text { [BU] }\end{array}$ & $\begin{array}{c}\text { Water } \\
\text { absor } \\
\text { ption } \\
\%\end{array}$ & $\begin{array}{c}\text { Water } \\
\text { absorption } \\
\text { (corrected } \\
\text { for } 500 \text { BU) }\end{array}$ & $\begin{array}{c}\text { Water } \\
\text { absorption } \\
\text { (corrected } \\
\text { for } 14 \% \text { ) }\end{array}$ & $\begin{array}{l}\text { Dev. } \\
\text { time } \\
\text { (min) }\end{array}$ & $\begin{array}{l}\text { Stability } \\
\text { (min) }\end{array}$ & $\begin{array}{l}\text { Mixing } \\
\text { Tolerance } \\
\text { Index } \\
\text { (BU) }\end{array}$ & $\begin{array}{l}\text { Time of } \\
\text { break } \\
\text { down } \\
(\text { min })\end{array}$ & $\begin{array}{c}\text { Farinograph } \\
\text { quality number } \\
\text { (FQN) }\end{array}$ \\
\hline Control (00:100) & 498.75 & 85.18 & 85.08 & 79.58 & 6.78 & 3.98 & 31.75 & 11.68 & 116.75 \\
\hline SDRSF 10:90 & 500.25 & 80.43 & 80.43 & 75.93 & 5.43 & 2.25 & 53.00 & 7.90 & 79.93 \\
\hline SDRSF 15:85 & 501.75 & 79.58 & 79.58 & 75.08 & 5.28 & 2.40 & 56.75 & 7.18 & 71.75 \\
\hline SDRSF 20:80 & 505.25 & 78.33 & 78.53 & 73.93 & 5.05 & 2.00 & 70.25 & 6.53 & 65.25 \\
\hline SDRSF 25:75 & 511.00 & 77.98 & 78.28 & 73.48 & 4.88 & 1.23 & 100.00 & 6.08 & 60.00 \\
\hline $\mathrm{SE}( \pm)$ & 0.53 & 0.05 & 0.05 & 0.04 & 0.05 & 0.04 & 0.41 & 0.05 & 0.41 \\
\hline CD at5 (\%) & 1.60 & 0.14 & 0.15 & 0.14 & 0.13 & 0.11 & 1.25 & 0.16 & 1.24 \\
\hline CV (\%) & 0.21 & 0.12 & 0.12 & 0.13 & 1.63 & 3.18 & 1.32 & 1.35 & 1.05 \\
\hline
\end{tabular}

Each value is an average of four determinations. SDRSF $=$ Sprouted Decorticated Roselle Seed Flour 
Table.9 Effects of addition of whole Roselle seeds flour (WRSF) on Energy [ $\left.\mathrm{cm}^{2}\right]$

\begin{tabular}{|l|c|c|c|}
\hline \multirow{2}{*}{$\begin{array}{l}\text { Treatments } \\
\text { Roselle seed flour:wheat }\end{array}$} & \multicolumn{3}{|c|}{ Proving Time (min) } \\
\hline Control (00:100) & 30 & 60 & 90 \\
\hline WRSF 10:90 & 34.75 & 34.75 & 31.75 \\
\hline WRSF 15:85 & 26.75 & 29.25 & 22.00 \\
\hline WRSF 20:80 & 15.50 & 15.25 & 16.25 \\
\hline WRSF 25:75 & 12.50 & 13.75 & 12.75 \\
\hline SE( $\mathbf{( )}$ & 0.57 & 0.48 & 0.56 \\
\hline CD at5 (\%) & 1.72 & 1.44 & 1.70 \\
\hline CV (\%) & 4.47 & 4.07 & 5.43 \\
\hline
\end{tabular}

Each value is an average of four determinations. WRSF $=$ Whole Roselle seeds flour

Table.10 Effects of addition of whole Roselle seeds flour (WRSF) on Resistance to Extension [BU]

\begin{tabular}{|l|c|c|c|}
\hline \multirow{2}{*}{$\begin{array}{l}\text { Treatments } \\
\text { Roselle seed flour:wheat }\end{array}$} & \multicolumn{3}{|c|}{ Proving Time (min) } \\
\hline Control (00:100) & 30 & 60 & 90 \\
\hline WRSF 10:90 & 273.00 & 185.00 & 165.25 \\
\hline WRSF 15:85 & 199.00 & 174.75 & 162.50 \\
\hline WRSF 20:80 & 120.75 & 120.25 & 129.25 \\
\hline WRSF 25:75 & 97.75 & 97.00 & 94.75 \\
\hline SE( $)$ & 0.64 & 0.45 & 0.48 \\
\hline CD at5 (\%) & 1.92 & 1.36 & 1.40 \\
\hline CV (\%) & 0.64 & 0.54 & 0.60 \\
\hline
\end{tabular}

Each value is an average of four determinations. WRSF $=$ Whole Roselle seeds flour

Table.11 Effects of addition of whole Roselle seeds flour (WRSF) on Extensibility [mm]

\begin{tabular}{|l|c|c|c|}
\hline \multirow{2}{*}{$\begin{array}{l}\text { Treatments } \\
\text { Roselle seed flour:wheat }\end{array}$} & \multicolumn{3}{|c|}{ Proving Time (min) } \\
\hline Control (00:100) & 30 & 60 & 90 \\
\hline WRSF 10:90 & 88.75 & 89.75 & 81.75 \\
\hline WRSF 15:85 & 87.00 & 86.00 & 79.00 \\
\hline WRSF 20:80 & 82.75 & 84.75 & 77.75 \\
\hline WRSF 25:75 & 81.75 & 75.75 & 70.75 \\
\hline SE( $)$ & 0.50 & 0.45 & 0.56 \\
\hline CD at5 (\%) & 1.52 & 1.36 & 1.70 \\
\hline CV (\%) & 1.18 & 1.09 & 1.46 \\
\hline
\end{tabular}

Each value is an average of four determinations. $\mathrm{WRSF}=$ Whole Roselle seeds flour 
Table.12 Effects of addition of whole Roselle seeds flour (WRSF) on Maximum [BU]

\begin{tabular}{|l|c|c|c|}
\hline \multirow{2}{*}{$\begin{array}{l}\text { Treatments } \\
\text { Roselle seed flour:wheat }\end{array}$} & \multicolumn{3}{|c|}{ Proving Time (min) } \\
\cline { 2 - 4 } Control (00:100) & 30 & 60 & 90 \\
\hline WRSF 10:90 & 275.00 & 185.50 & 173.25 \\
\hline WRSF 15:85 & 211.75 & 181.75 & 168.00 \\
\hline WRSF 20:80 & 133.25 & 149.75 & 151.25 \\
\hline WRSF 25:75 & 120.75 & 122.75 & 124.75 \\
\hline SE( $\mathbf{( )}$ & 0.47 & 0.52 & 0.53 \\
\hline CD at5 (\%) & 1.40 & 1.56 & 1.60 \\
\hline CV (\%) & 0.44 & 0.56 & 0.60 \\
\hline
\end{tabular}

Each value is an average of four determinations. WRSF $=$ Whole Roselle seeds flour

Table.13 Effects of addition of whole Roselle Seeds flour (WRSF) on Ratio Number

\begin{tabular}{|l|c|c|c|}
\hline \multirow{2}{*}{ Treatments } & \multicolumn{3}{|c|}{ Proving Time (min) } \\
\hline Roselle seed flour:wheat & 30 & 60 & 90 \\
\hline Control (00:100) & 3.58 & 3.08 & 3.18 \\
\hline WRSF 10:90 & 2.93 & 1.95 & 1.93 \\
\hline WRSF 15:85 & .38 & 1.88 & 1.88 \\
\hline WRSF 20:80 & 1.33 & 1.53 & 1.53 \\
\hline WRSF 25:75 & 1.28 & 1.38 & 1.38 \\
\hline SE(土) & 0.04 & 0.05 & 0.04 \\
\hline CD at5 (\%) & 0.14 & 0.15 & 0.14 \\
\hline CV (\%) & 4.17 & 5.27 & 4.85 \\
\hline
\end{tabular}

Each value is an average of four determinations. WRSF $=$ Whole Roselle seeds flour

Table.14 Effects of addition of whole Roselle seeds flour (WRSF) on Ratio Number [Max.]

\begin{tabular}{|l|c|c|c|}
\hline \multirow{2}{*}{$\begin{array}{l}\text { Treatments } \\
\text { Roselle seed flour:wheat }\end{array}$} & \multicolumn{3}{|c|}{ Proving Time (min) } \\
\hline Control (00:100) & 30 & 60 & 90 \\
\hline WRSF 10:90 & 3.68 & 3.18 & 3.38 \\
\hline WRSF 15:85 & 3.03 & 2.03 & 2.03 \\
\hline WRSF 20:80 & 2.58 & 1.80 & 1.78 \\
\hline WRSF 25:75 & 1.53 & 1.63 & 1.53 \\
\hline SE( $)$ & 1.40 & 1.58 & 1.33 \\
\hline CD at5 (\%) & 0.05 & 0.06 & 0.06 \\
\hline CV (\%) & 0.14 & 0.17 & 0.19 \\
\hline
\end{tabular}

Each value is an average of four determinations. WRSF $=$ Whole Roselle seeds flour 
Table.15 Effects of addition of decorticated Roselle seeds flour (DRSF) on Energy [ $\left.\mathrm{cm}^{2}\right]$

\begin{tabular}{|l|c|c|c|}
\hline \multirow{2}{*}{ Treatments } & \multicolumn{3}{|c|}{ Proving Time (min) } \\
\hline Roselle seed flour:wheat & 30 & 60 & 90 \\
\hline Control (00:100) & 38.75 & 34.75 & 31.75 \\
\hline DRSF 10:90 & 20.00 & 19.00 & 16.75 \\
\hline DRSF 15:85 & 17.75 & 17.25 & 14.75 \\
\hline DRSF 20:80 & 12.00 & 10.25 & 9.25 \\
\hline DRSF 25:75 & 10.75 & 9.75 & 8.75 \\
\hline SE(土) & 0.55 & 0.47 & 0.48 \\
\hline CD at5 (\%) & 1.66 & 1.40 & 1.44 \\
\hline CV (\%) & 5.56 & 5.12 & 5.89 \\
\hline
\end{tabular}

Each value is an average of four determinations. DRSF $=$ Decorticated Roselle seeds flour

Table 16 Effects of addition of decorticated Roselle seeds flour (DRSF) on Resistance to Extension [BU]

\begin{tabular}{|l|c|c|c|}
\hline \multirow{2}{*}{ Treatments } & \multicolumn{3}{|c|}{ Proving Time (min) } \\
\hline Roselle seed flour:wheat & 30 & 60 & 90 \\
\hline Control (00:100) & 304.75 & 263.75 & 251.75 \\
\hline DRSF 10:90 & 144.00 & 119.25 & 129.00 \\
\hline DRSF 15:85 & 119.75 & 117.25 & 109.75 \\
\hline DRSF 20:80 & 77.25 & 70.75 & 63.25 \\
\hline DRSF 25:75 & 71.75 & 62.75 & 54.75 \\
\hline SE( $)$ & 0.47 & 0.48 & 0.47 \\
\hline CD at5 (\%) & 1.40 & 1.44 & 1.40 \\
\hline CV (\%) & 0.65 & 0.76 & 0.76 \\
\hline
\end{tabular}

Each value is an average of four determinations. DRSF $=$ Decorticated Roselle seeds flour

Table.17 Effects of addition of decorticated Roselle seeds flour (DRSF) on Extensibility [mm]

\begin{tabular}{|l|c|c|c|}
\hline Treatments & \multicolumn{3}{|c|}{ Proving Time (min) } \\
\hline Roselle seed flour:wheat & 30 & 60 & 90 \\
\hline Control (00:100) & 88.75 & 89.75 & 81.75 \\
\hline DRSF 10:90 & 87.00 & 83.00 & 74.25 \\
\hline DRSF 15:85 & 85.00 & 78.25 & 72.00 \\
\hline DRSF 20:80 & 75.25 & 74.25 & 67.25 \\
\hline DRSF 25:75 & 72.75 & 65.75 & 65.75 \\
\hline SE( () & 0.45 & 0.56 & 0.56 \\
\hline CD at5 (\%) & 1.36 & 1.70 & 1.69 \\
\hline CV (\%) & 1.10 & 1.44 & 1.56 \\
\hline
\end{tabular}

Each value is an average of four determinations. DRSF $=$ Decorticated Roselle seeds flour 
Table.18 Effects of addition of decorticated Roselle seeds flour (DRSF) on Maximum [BU]

\begin{tabular}{|l|c|c|c|}
\hline Treatments & \multicolumn{3}{|c|}{ Proving Time (mm) } \\
\cline { 2 - 4 } Roselle seed flour:wheat & 30 & 60 & 90 \\
\hline Control (00:100) & 317.75 & 277.75 & 270.75 \\
\hline DRSF 10:90 & 165.25 & 152.25 & 157.25 \\
\hline DRSF 15:85 & 140.00 & 144.00 & 140.00 \\
\hline DRSF 20:80 & 112.25 & 105.25 & 102.00 \\
\hline DRSF 25:75 & 111.00 & 104.00 & 100.00 \\
\hline SE( $\mathbf{( )}$ & 0.71 & 0.64 & 0.54 \\
\hline CD at5 (\%) & 2.14 & 1.92 & 1.63 \\
\hline CV (\%) & 0.84 & 0.81 & 0.70 \\
\hline
\end{tabular}

Each value is an average of four determinations. DRSF $=$ Decorticated Roselle seeds flour

Table.19 Effects of addition of decorticated Roselle seeds flour (DRSF) on Ratio Number

\begin{tabular}{|l|c|c|c|}
\hline Treatments & \multicolumn{3}{|c|}{ Proving Time (min) } \\
\cline { 2 - 4 } Roselle seed flour:wheat & 30 & 60 & 90 \\
\hline Control (00:100) & 3.58 & 3.08 & 3.18 \\
\hline DRSF 10:90 & 1.63 & 1.60 & 1.70 \\
\hline DRSF 15:85 & 1.48 & 1.48 & 1.58 \\
\hline DRSF 20:80 & 1.13 & 0.83 & 0.73 \\
\hline DRSF 25:75 & 1.08 & 0.63 & 0.68 \\
\hline SE( $\mathbf{( )}$ & 0.05 & 0.06 & 0.04 \\
\hline CD at5 (\%) & 0.14 & 0.17 & 0.14 \\
\hline CV (\%) & 5.39 & 7.40 & 5.93 \\
\hline
\end{tabular}

Each value is an average of four determinations. DRSF $=$ Decorticated Roselle seeds flour

Table.20 Effects of addition of decorticated Roselle seeds flour (DRSF) on Ratio Number [Max]

\begin{tabular}{|l|c|c|c|}
\hline Treatments & \multicolumn{3}{|c|}{ Proving Time (min) } \\
\cline { 2 - 4 } Roselle seed flour:wheat & 30 & 60 & 90 \\
\hline Control (00:100) & 3.68 & 3.18 & 3.38 \\
\hline DRSF 10:90 & 1.83 & 1.93 & 2.03 \\
\hline DRSF 15:85 & 1.78 & 1.80 & 1.80 \\
\hline DRSF 20:80 & 1.50 & 1.43 & 1.43 \\
\hline DRSF 25:75 & 1.38 & 1.38 & 1.38 \\
\hline SE( $\mathbf{( )}$ & 0.05 & 0.04 & 0.06 \\
\hline CD at5 (\%) & 0.14 & 0.14 & 0.17 \\
\hline CV $(\%)$ & 4.59 & 4.80 & 5.63 \\
\hline
\end{tabular}

Each value is an average of four determinations. DRSF $=$ Decorticated Roselle seeds flour 
Table.21 Effects of addition of sprouted whole Roselle seeds flour (SWRSF) on Energy $\left[\mathrm{cm}^{2}\right]$

\begin{tabular}{|l|c|c|c|}
\hline \multirow{2}{*}{$\begin{array}{l}\text { Treatments } \\
\text { Roselle seed flour:wheat }\end{array}$} & \multicolumn{3}{|c|}{ Proving Time (min) } \\
\hline Control (00:100) & 30 & 60 & 90 \\
\hline SWRSF 10:90 & 38.75 & 34.75 & 31.75 \\
\hline SWRSF 15:85 & 25.25 & 24.00 & 26.25 \\
\hline SWRSF 20:80 & 19.75 & 18.75 & 20.00 \\
\hline SWRSF 25:75 & 17.00 & 16.25 & 17.25 \\
\hline SE(土) & 14.75 & 14.75 & 16.75 \\
\hline CD at5 (\%) & 0.47 & 0.46 & 0.47 \\
\hline CV (\%) & 1.40 & 1.39 & 1.41 \\
\hline
\end{tabular}

Each value is an average of four determinations. SWRSF $=$ Sprouted whole Roselle seeds flour

Table.22 Effects of addition of sprouted whole Roselle seeds flour (SWRSF) on Resistance to Extension [BU]

\begin{tabular}{|l|c|c|c|}
\hline \multirow{2}{*}{$\begin{array}{l}\text { Treatments } \\
\text { Roselle seed flour:wheat }\end{array}$} & \multicolumn{3}{|c|}{ Proving Time (min) } \\
\hline Control (00:100) & 30 & 60 & 90 \\
\hline SWRSF 10:90 & 193.00 & 190.00 & 251.75 \\
\hline SWRSF 15:85 & 146.75 & 140.75 & 196.00 \\
\hline SWRSF 20:80 & 126.75 & 124.75 & 149.00 \\
\hline SWRSF 25:75 & 114.75 & 117.75 & 126.75 \\
\hline SE( \pm ()) & 0.56 & 0.47 & 0.45 \\
\hline CD at5 (\%) & 1.70 & 1.40 & 1.36 \\
\hline CV (\%) & 0.64 & 1.56 & 1.51 \\
\hline
\end{tabular}

Each value is an average of four determinations. SWRSF $=$ Sprouted whole Roselle seeds flour

Table.23 Effects of addition of sprouted whole Roselle seeds flour (SWRSF) on Extensibility [mm]

\begin{tabular}{|l|c|c|c|}
\hline \multirow{2}{*}{$\begin{array}{l}\text { Treatments } \\
\text { Roselle seed flour:wheat }\end{array}$} & \multicolumn{3}{|c|}{ Proving Time (min) } \\
\hline Control (00:100) & 30 & 60 & 90 \\
\hline SWRSF 10:90 & 88.75 & 89.75 & 81.75 \\
\hline SWRSF 15:85 & 84.00 & 83.00 & 79.00 \\
\hline SWRSF 20:80 & 76.25 & 73.00 & 72.75 \\
\hline SWRSF 25:75 & 72.75 & 68.25 & 70.25 \\
\hline SE( $)$ & 0.45 & 67.75 & 68.75 \\
\hline CD at5 (\%) & 1.36 & 1.36 & 0.47 \\
\hline CV (\%) & 1.11 & 1.18 & 1.40 \\
\hline
\end{tabular}

Each value is an average of four determinations. SWRSF $=$ Sprouted whole Roselle seeds flour 
Table.24 Effects of addition of sprouted whole Roselle seeds flour (SWRSF) on Maximum [BU]

\begin{tabular}{|l|c|c|c|}
\hline \multirow{2}{*}{$\begin{array}{l}\text { Treatments } \\
\text { Roselle seed flour:wheat }\end{array}$} & \multicolumn{3}{|c|}{ Proving Time (min) } \\
\cline { 2 - 4 } Control (00:100) & 30 & 60 & 90 \\
\hline SWRSF 10:90 & 317.75 & 277.75 & 270.75 \\
\hline SWRSF 15:85 & 12.25 & 203.00 & 206.00 \\
\hline SWRSF 20:80 & 162.75 & 172.75 & 191.00 \\
\hline SWRSF 25:75 & 158.00 & 170.25 & 178.25 \\
\hline SE( $\mathbf{( )}$ & 0.47 & 162.75 & 176.75 \\
\hline CD at5 (\%) & 1.40 & 1.70 & 0.45 \\
\hline CV (\%) & 0.46 & 0.57 & 1.36 \\
\hline
\end{tabular}

Each value is an average of four determinations. SWRSF = Sprouted whole Roselle seeds flour

Table.25 Effects of addition of sprouted whole Roselle seeds flour (SWRSF) on Ratio Number

\begin{tabular}{|l|c|c|c|}
\hline \multirow{2}{*}{$\begin{array}{l}\text { Treatments } \\
\text { Roselle seed flour:wheat }\end{array}$} & \multicolumn{3}{|c|}{ Proving Time (min) } \\
\cline { 2 - 4 } Control (00:100) & 30 & 60 & 90 \\
\hline SWRSF 10:90 & 3.58 & 3.08 & 3.18 \\
\hline SWRSF 15:85 & 2.20 & 2.23 & 2.40 \\
\hline SWRSF 20:80 & 1.88 & 2.08 & 2.28 \\
\hline SWRSF 25:75 & 1.63 & 1.83 & 2.03 \\
\hline SE( $)$ & 1.58 & 1.78 & 1.88 \\
\hline CD at5 (\%) & 0.05 & 0.04 & 0.05 \\
\hline CV (\%) & 0.14 & 0.13 & 0.14 \\
\hline
\end{tabular}

Each value is an average of four determinations. SWRSF $=$ Sprouted whole Roselle seeds flour

Table.26 Effects of addition of sprouted whole Roselle seeds flour (SWRSF) on Ratio Number [Max.]

\begin{tabular}{|l|c|c|c|}
\hline \multirow{2}{*}{$\begin{array}{l}\text { Treatments } \\
\text { Roselle seed flour:wheat }\end{array}$} & \multicolumn{3}{|c|}{ Proving Time (min) } \\
\hline Control (00:100) & 30 & 60 & 90 \\
\hline SWRSF 10:90 & 3.68 & 3.18 & 3.38 \\
\hline SWRSF 15:85 & 2.43 & 2.43 & 2.70 \\
\hline SWRSF 20:80 & 2.28 & 2.38 & 2.58 \\
\hline SWRSF 25:75 & 2.10 & 2.10 & 2.23 \\
\hline SE( $\mathbf{( )}$ & 1.98 & 2.08 & 2.08 \\
\hline CD at5 (\%) & 0.05 & 0.05 & 0.04 \\
\hline CV (\%) & 0.16 & 0.16 & 0.14 \\
\hline
\end{tabular}

Each value is an average of four determinations. SWRSF $=$ Sprouted whole Roselle seeds flour 
Table.27 Effects of addition of sprouted decorticated Roselle seeds flour (SDRSF) on Energy $\left[\mathrm{cm}^{2}\right]$

\begin{tabular}{|l|c|c|c|}
\hline Treatments & \multicolumn{3}{|c|}{ Proving Time (min) } \\
\cline { 2 - 4 } Roselle seed flour:wheat & 30 & 60 & 90 \\
\hline Control (00:100) & 38.75 & 34.75 & 31.75 \\
\hline SDRSF 10:90 & 24.00 & 23.00 & 23.00 \\
\hline SDRSF 15:85 & 20.75 & 18.00 & 16.50 \\
\hline SDRSF 20:80 & 12.50 & 10.25 & 10.00 \\
\hline SDRSF 25:75 & 9.75 & 8.75 & 8.75 \\
\hline SE( $($ ) & 0.47 & 0.45 & 0.42 \\
\hline CD at5 (\%) & 1.42 & 1.36 & 1.26 \\
\hline CV (\%) & 4.44 & 4.77 & 4.65 \\
\hline
\end{tabular}

Each value is an average of four determinations. SDRSF $=$ Sprouted decorticated Roselle seeds flour

Table.28 Effects of addition of sprouted decorticated Roselle seeds flour (SDRSF) on Resistance to Extension [BU]

\begin{tabular}{|l|c|c|c|}
\hline Treatments & \multicolumn{3}{|c|}{ Proving Time (min) } \\
\cline { 2 - 4 } Roselle seed flour:wheat & 30 & 60 & 90 \\
\hline Control (00:100) & 304.75 & 263.75 & 251.75 \\
\hline SDRSF 10:90 & 170.00 & 167.00 & 170.00 \\
\hline SDRSF 15:85 & 141.00 & 129.25 & 127.00 \\
\hline SDRSF 20:80 & 90.50 & 79.00 & 72.75 \\
\hline SDRSF 25:75 & 60.75 & 49.75 & 45.75 \\
\hline SE( $\mathbf{( )}$ & 0.49 & 0.41 & 0.45 \\
\hline CD at5 (\%) & 1.48 & 1.24 & 1.36 \\
\hline CV (\%) & 0.64 & 0.60 & 0.68 \\
\hline
\end{tabular}

Each value is an average of four determinations. SDRSF $=$ Sprouted decorticated Roselle seeds flour

Table.29 Effects of addition of sprouted decorticated Roselle seeds flour (SDRSF) on Extensibility [mm]

\begin{tabular}{|l|c|c|c|}
\hline \multirow{2}{*}{$\begin{array}{l}\text { Treatments } \\
\text { Roselle seed flour:wheat }\end{array}$} & \multicolumn{3}{|c|}{ Proving Time (min) } \\
\cline { 2 - 4 } Control (00:100) & 88.75 & 60 & 90 \\
\hline SDRSF 10:90 & 93.25 & 88.75 & 81.75 \\
\hline SDRSF 15:85 & 92.75 & 87.00 & 79.00 \\
\hline SDRSF 20:80 & 84.25 & 70.25 & 77.75 \\
\hline SDRSF 25:75 & 71.25 & 61.00 & 61.00 \\
\hline SE( $\mathbf{( )}$ & 0.48 & 0.64 & 59.75 \\
\hline CD at5 (\%) & 1.44 & 1.92 & 0.45 \\
\hline CV (\%) & 1.11 & 1.60 & 1.36 \\
\hline
\end{tabular}

Each value is an average of four determinations. SDRSF $=$ Sprouted decorticated Roselle seeds flour 
Table.30 Effects of addition of sprouted decorticated Roselle seeds flour (SDRSF) on Maximum [BU]

\begin{tabular}{|l|c|c|c|}
\hline Treatments & \multicolumn{3}{|c|}{ Proving Time (min) } \\
\cline { 2 - 4 } Roselle seed flour:wheat & 30 & 60 & 90 \\
\hline Control (00:100) & 317.75 & 277.75 & 270.75 \\
\hline SDRSF 10:90 & 184.00 & 189.00 & 185.25 \\
\hline SDRSF 15:85 & 163.00 & 148.25 & 151.75 \\
\hline SDRSF 20:80 & 123.75 & 124.00 & 129.25 \\
\hline SDRSF 25:75 & 107.75 & 109.75 & 115.75 \\
\hline SE( $\mathbf{( )}$ & 0.45 & 0.49 & 0.47 \\
\hline CD at5 (\%) & 1.36 & 1.47 & 1.44 \\
\hline CV (\%) & 0.50 & 0.57 & 0.56 \\
\hline
\end{tabular}

Each value is an average of four determinations. SDRSF $=$ Sprouted decorticated Roselle seeds flour

Table.31 Effects of addition of sprouted decorticated Roselle seeds flour (SDRSF) on Ratio Number

\begin{tabular}{|l|c|c|c|}
\hline Treatments & \multicolumn{3}{|c|}{ Proving Time (min) } \\
\cline { 2 - 4 } Roselle seed flour:wheat & 30 & 60 & 90 \\
\hline Control (00:100) & 3.58 & 3.08 & 3.18 \\
\hline SDRSF 10:90 & 1.78 & 1.83 & 2.00 \\
\hline SDRSF 15:85 & 1.58 & 1.58 & 1.68 \\
\hline SDRSF 20:80 & 1.10 & 1.03 & 1.13 \\
\hline SDRSF 25:75 & 0.88 & 0.88 & 0.88 \\
\hline SE( $\mathbf{( )}$ & 0.05 & 0.04 & 0.05 \\
\hline CD at5 (\%) & 0.15 & 0.14 & 0.14 \\
\hline CV (\%) & 5.62 & 5.72 & 5.26 \\
\hline
\end{tabular}

Each value is an average of four determinations. SDRSF $=$ Sprouted decorticated Roselle seeds flour

Table.32 Effects of addition of sprouted decorticated Roselle seeds flour (SDRSF) on Ratio Number [Max]

\begin{tabular}{|l|c|c|c|}
\hline Treatments & \multicolumn{3}{|c|}{ Proving Time (min) } \\
\hline Roselle seed flour:wheat & 30 & 60 & 90 \\
\hline Control (00:100) & 3.68 & 3.18 & 3.38 \\
\hline SDRSF 10:90 & 1.93 & 2.03 & 2.13 \\
\hline SDRSF 15:85 & 1.88 & 1.85 & 2.08 \\
\hline SDRSF 20:80 & 1.53 & 1.70 & 1.83 \\
\hline SDRSF 25:75 & 1.48 & 1.58 & 1.70 \\
\hline SE( $)$ & 0.05 & 0.04 & 0.05 \\
\hline CD at5 (\%) & 0.14 & 0.13 & 0.14 \\
\hline CV (\%) & 4.57 & 4.19 & 4.18 \\
\hline
\end{tabular}

Each value is an average of four determinations. SDRSF $=$ Sprouted decorticated Roselle seeds flour 
Fig.1 Amylogram of dough prepared with 100\% Wheat flour (Control).

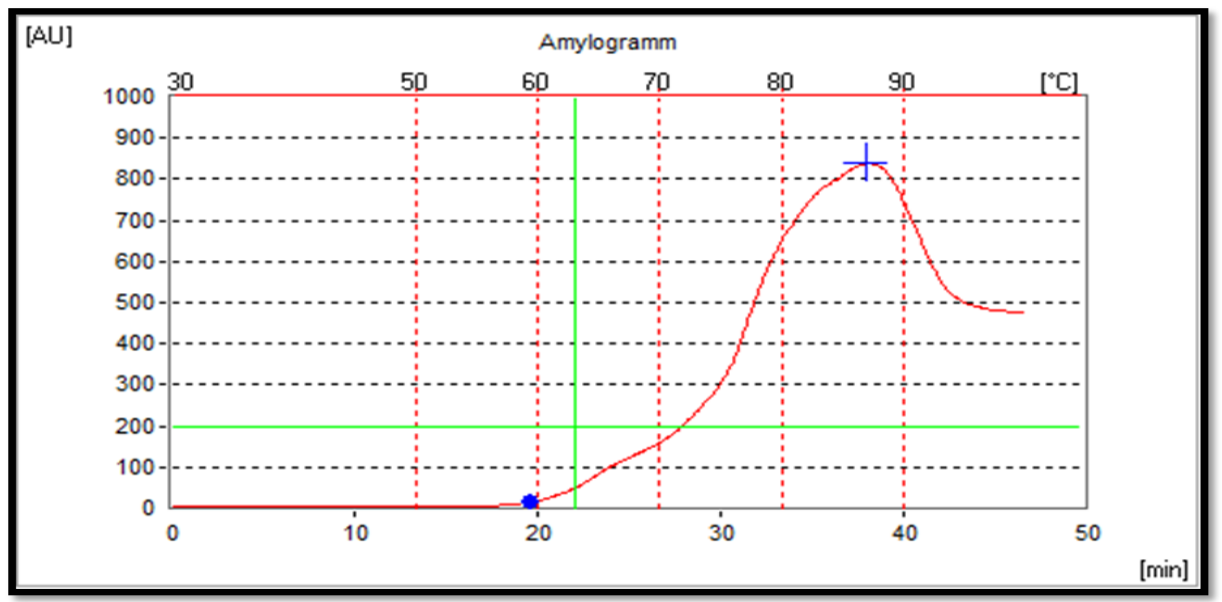

Fig.2 Amylogram of dough prepared with WRSF 15:85 composite flour

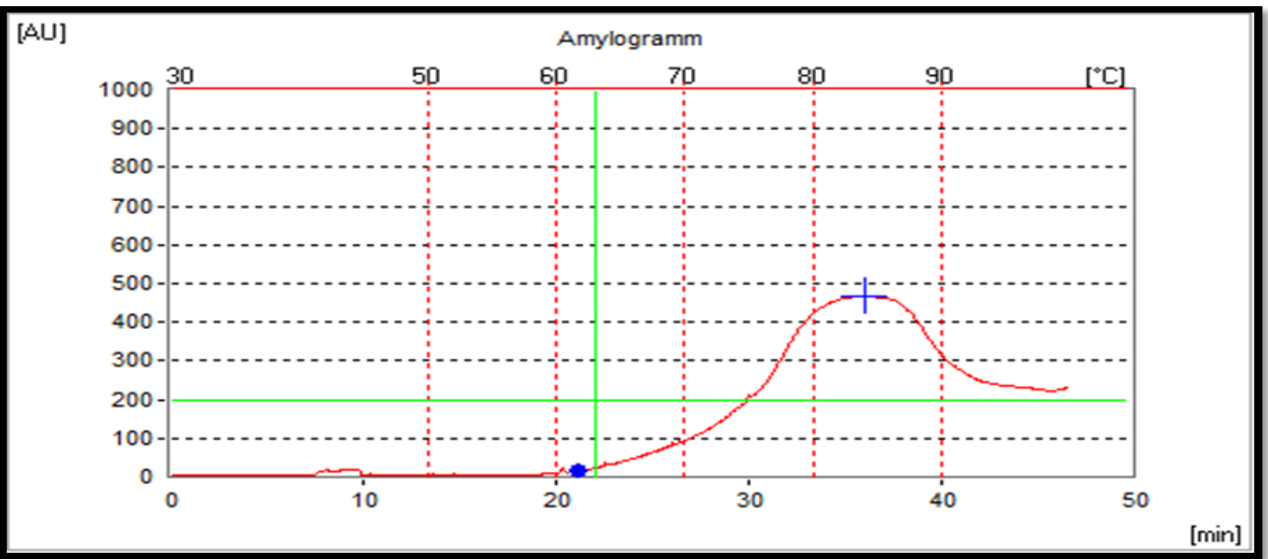

Fig.3 Amylogram of dough prepared with DRSF 15:85 composite flour

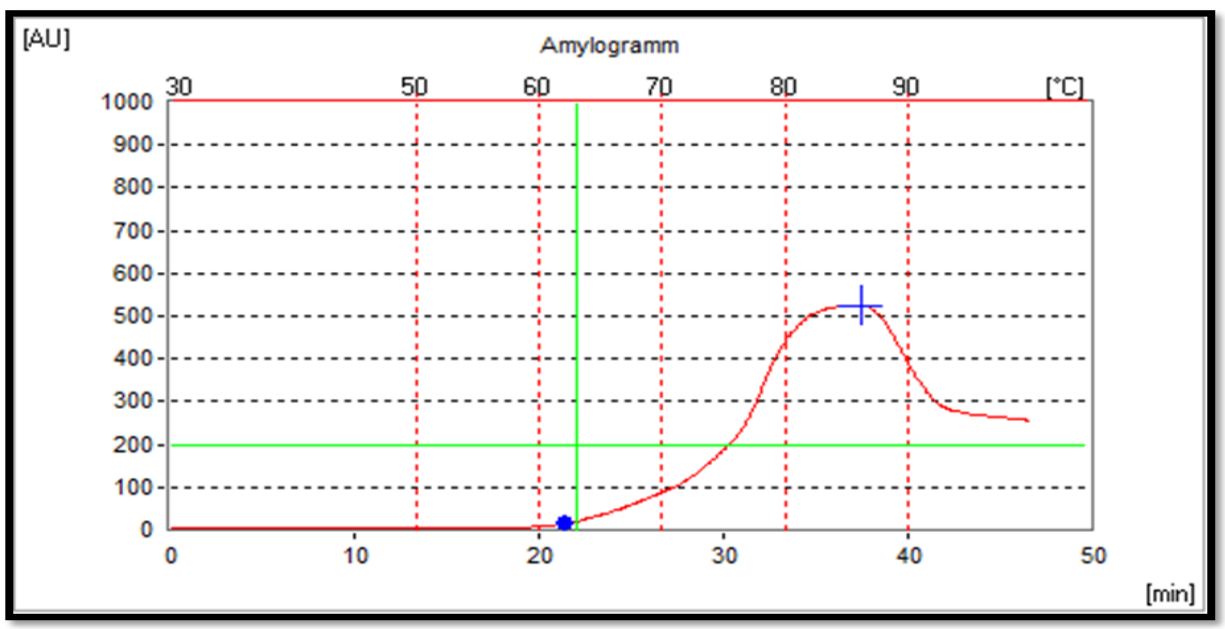


Fig.4 Amylogram of dough prepared with SWRSF 15:85 composite flour

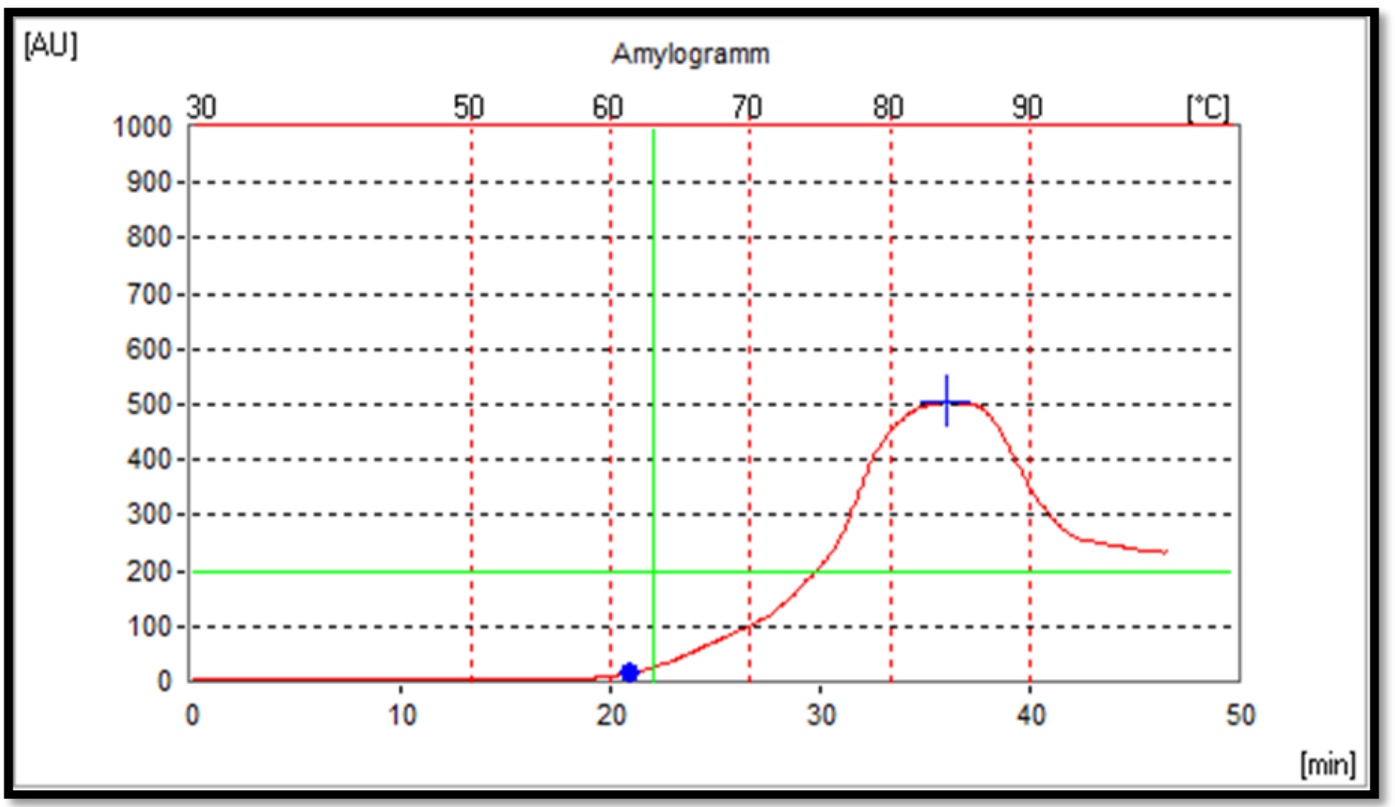

Fig.5 Amylogram of dough prepared with SDRSF 15:85 composite flour.

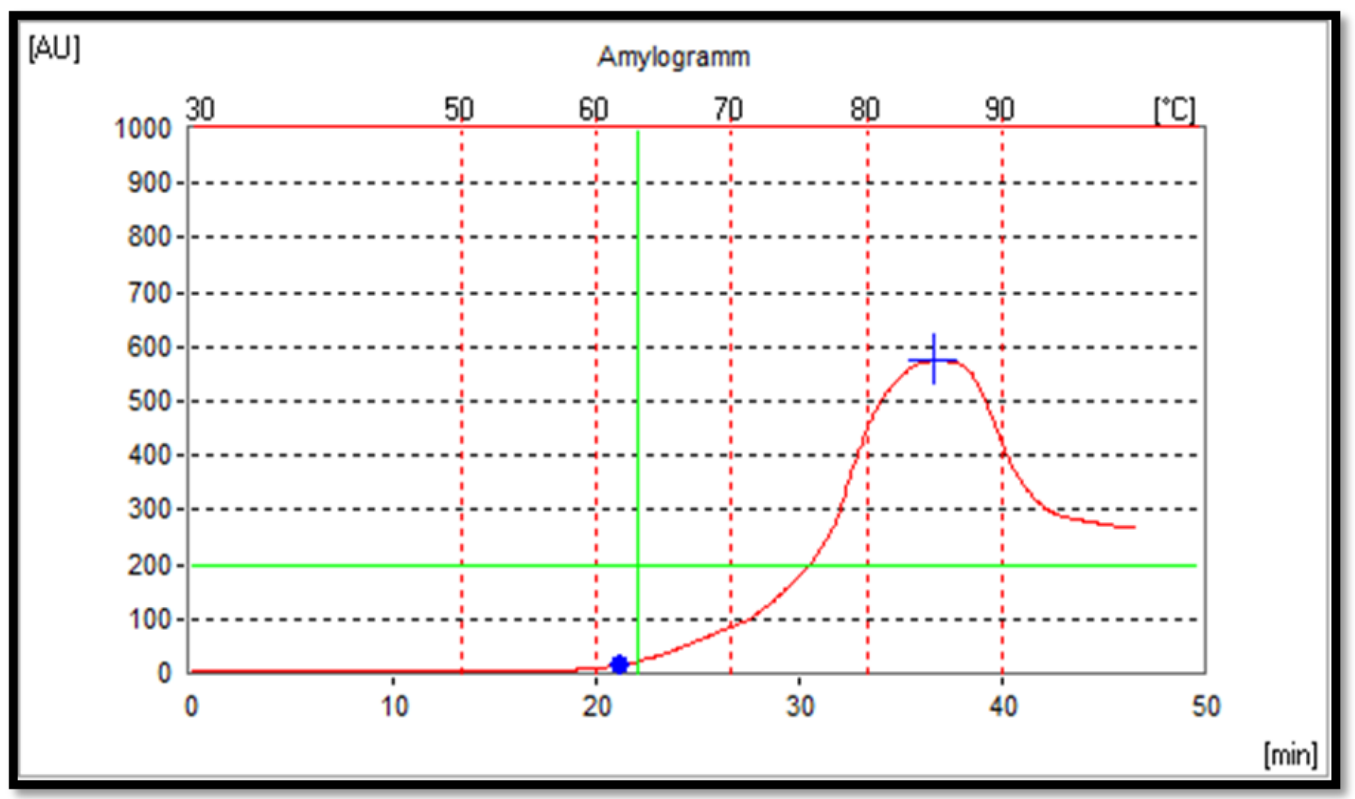


For sprouted whole Roselle seed flour (SWRSF), the farinogram results categorized $10 \%, 15 \%, 20 \%$ and $25 \%$ as strong and medium flour respectively (Preston, 1984), with $10 \%, 15 \%$ and $20 \%$ compared favourably with control as strong flours. Having MTI values of 42.0, 44.25, 45.0 and 31.75 BU, development time 5.60, 6.08, 5.65 and $6.78 \mathrm{~min}$, and water absorption 76.73 , $74.93,72.95$ and $79.58 \%$ respectively (Table $7)$. There is a significant difference $(\mathrm{P}<0.05)$ between the observed values of dough characteristic of all the treatment formulations. Water absorption, development time, stability and farinograph quality number (FQN) values decreased with the inclusion of SWRSF in the composite mixture, with the exception of MTI [BU] showing an inverse relationship.

For sprouted decorticated Roselle seed flour (SDRSF), the farinogram defined and categorized $10 \%, 15 \%, 20 \%$ and $25 \%$ as strong, medium and weak flours respectively (Preston, 1984), with 10\% compared fovourably with control as strong flours. Having MTI values of 53.0 and 31.75 BU, development time 5.43 and $6.78 \mathrm{~min}$, and water absorption 75.93 and $79.58 \%$ respectively (Table 8 ). There is a significance difference $(\mathrm{P}<0.05)$ between the observed values of dough characteristic of all the treatment formulations. Water absorption, Development time, stability, and farinograph quality number (FQN) values decreased with the inclusion of SDRSF in the composite mixture, with the exception of MTI [BU] showing an inverse relationship.

Farinograph results of both whole seed flour treatments showed better superior functional properties compared to their decorticated counterparts, in terms of stability and mixing tolerance index which are indications of dough strength and the degree of softening during mixing (AACC, 2000 Method 54-21).
Whole seed flour treatments are more fiber rich (El-Adawy and Khalil, 1994; Ismail et al., 2008) as such that could improve significantly the flour functional properties.

This study provides sufficient results on water absorption and dough development properties of Roselle seeds flour in composite formulation with wheat; results may serve as a guide to bakers in its utilization as an enriched ingredient in value added bakery products.

\section{Extensograph studies}

The extensograph technique was used to study the rheological properties of the mixture flour of Roselle seed flour and wheat flour used for the preparation of bakery products such as cookies. Under this study energy required, resistance to extension, extensibility, maximum [BU], ratio number and ratio number [Max] index parameters were studied. Extensograph results of wheat flour (control), whole Roselle seed flour (Tables 9-14), decorticated Roselle seed flour (Tables 1520); sprouted whole Roselle seed flour (Tables 21-26 and sprouted decorticated Roselle seed flour (Tables 27-32) gives good idea for their utilization in various baker products.

The results of extensograph indicated that decorticated without sprouting and after sprouting Roselle seed flour gave good results for better quality of the bakery products such as cookies. However, the decorticated flour sample showed slightly lower values than the whole seed flour for all above extensograph observations. There is no any literature on these aspects for supporting these results.

Results of preliminary studies on pretreatments had considered sprouted seed treatments as the best by virtue of it proximate and mineral composition, improved 
functional properties based on amylograph, farinograph and extensograph studies.

\section{References}

AACC. (2000). Amylograph, Farinograph and Extensograph, Approved methods of the American Association of Cereal Chemists (10th ed.). St. Paul, MN: Author (methods No. 22-10, 54-21 and 54-10

Bala, A., Gul, K. and Riar, C. S. (2015). Functional and sensory properties of cookies prepared from wheat flour supplemented with cassava and water chestnut flours. Cogent Food \& Agriculture, 1, 1019815.

Bambgoye, A.I. and Adejumo, O.I. (2009). Physical Properties of Roselle (Hibiscus sabdariffa L.) Seed. Agricultural Engineering International: the CIGR Ejournal. Manuscript 1154.Vol. XI.

El-Adawy, T.A. and Khalil, A.H. (1994). Characteristics of roselle seeds as a new source of protein and lipid. Journal of Agricultural and Food Chemistry 42, 1896-1900

Emmy Hainida, K.I., Amin, I., Normah, H., Norhaizan, M.E., and Ainul, Z. (2008). Effects of defatted dried roselle (Hibiscus sabdariffa L.) Seed powder on lipid profiles of hypercholesterolemia rats. J. Sci. Food Agric. 88: 1043-1050.

Ismail, A., Emmy Hainida, K.I. and Halimatul, S.M.N. (2008). Roselle (Hibiscus sabdariffa L.) seedsNutritional Composition, Protein Quality and Health Benefits, Food 2(1), 1-16.

Karma Bako Rimamcwe, Chavan, U. D., Kotecha, P. M. and Lande, S. B. (2019). Effects of addition of sprouted whole Roselle seeds flour on rheological properties. International Journal of Current Research. 9: (12): 4265-4269.
Karma Bako Rimamcwe and U.D. Chavan (2017d). Antioxidant Activity and Nutritional Value of Roselle Seeds Flour. Int. J. Curr. Microbiol. App. Sci. 6(4): 2654-2663.

Karma Bako Rimamcwe, Chavan, U. D., Dalvi, U. S. and Gaikwad, R. S. (2017c). Nutritional quality of Roselle seed flour cookies. International Journal of Current Research. 9: (12): 6305363058.

Karma Bako Rimamcwe, Chavan, U. D., Nimbalkar, C. A. and Kahar, S. P. (2017a). Rheometry of Roselle (Hibiscus Sabdariffa L.) seed oil. Int. J. Pure App. Biosci. 5 (2): 987-993.

Karma Bako Rimamcwe, Chavan, U. D., Pawar, G. H. and Gaikwad, R. S. (2017b). Nitrogen Solubility and Functional Properties of Roselle Seed Flour. Int. J. Curr. Microbiol. App. Sci. 6(8): 1131-1139.

Karma Bako Rimamcwe, U. D. Chavan, S. B. Lande, C. A. Nimbalkar and U. S. Dalvi. (2018a). Physical and sensory quality of Roselle seed flour cookies. Int. J. Adv. Res. Biol. Sci. 5: (5): 160171.

Karma Bako Rimamcwe, U.D. Chavan, Ashok Kadlag and V.S. Wani. (2018b). Effect of Addition of Roselle Seed Flour on Color and Textural Properties of Cookies. Int. J. Curr. Microbiol. App. Sci. 7 (4): 3726-3737.

Karma Bako Rimamcwe., Chavan, U. D., Kadlag, A. D., Todmal, S. M. and Wani, V. S. (2018c). Effect of addition of whole Roselle seed flour on rheological properties of whole wheat flour. International Journal of Current Research. 10 (6): 70848-70856.

Karma, B.R. and Chavan, U.D. (2016). Physical Properties and Nutritional Potentials of Indian Roselle (Hibiscus sabdariffa L.) Seeds. Int. J. of Cur. Res., 8(9): 38644-38648. 
Myfolia.com (2016), online source: https://myfolia.com

Nyam KL, Tan YN, Tan CP and Kamariah, L., (2012). In vitro Antioxidant Activities of Extract and Oil from Roselle (Hibiscus sabdariffa L.) Seed against Sunflower Oil Autoxidation, Mal J Nutri 18(2): 265-274.

Nyam, Kar-Lin; Leao, Sod-Ying; Tan, ChinPing; and Long, Kamariah (2014). Functional Properties of Roselle (Hibiscus sabdariffa L.) seed and its application as bakery product, J. Food Sci. Technol 51(12):3830-3837.

Nzikou, J.M.,Bouanga-Kalou, G., Latos, L., Ganongo-Po, F.B., MboungouMboussi, P.S., Moutoula, F.E., PanyooAkdowa, E., Silou, T.H., and Desobry, S. (2012). Characteristics and Nutritional Evaluation of Seed oil from Roselle (Hibiscus sabdariffa L.) in Congo-Brazzaville, Current Research Journal of Biological Sciences 3(2): 141-146.

Panse, V.G. and Sukhatme, P.V. (1967). Statistical methods for Agricultural Workers. $4^{\text {th }}$ ed., New Delhi
Platon, JF. (1997). Lipids in Cosmetology. Ol. Corps Gras Lipid; 4: 275-281.

Preston, K.R. (1984). Use of lyotropic salts to study the hydrophobic properties of wheat gluten proteins. Proc. Intl. Workshop on Gluten Proteins, $2^{\text {nd }}$, TNO, Wageningen.

Ramadan M.F. and Morsel J.T. (2004). Oxidative stability of black cumin (Nigella sativa L.), coriander (Coriandrum sativum L.) and niger (Guizotia abyssinica Cass.) crude seeAd oils upon stripping. Eur J Lipid Sci Technol; 106: 35-43.

Wani, S. H., Gull, A., Allaie, F., and Safapuri, T. A. (2015). Effects of incorporation of whey protein concentrate on physicochemical, texture, and microbial evaluation of developed cookies. Cogent Food \& Agriculture, 1, 1092406.

Yayock, J.Y., Lombin, G. and Owonubi, J.J. (1988) Crop Science and Production in Warm Climates. Macmillan Publishers, London, p.307.

\section{How to cite this article:}

Karma Bako Rimamcwe, U. D. Chavan and Kotecha, P. M. 2020. Rheological Properties of Roselle Seed Flour. Int.J.Curr.Microbiol.App.Sci. 9(07): 1856-1876. doi: https://doi.org/10.20546/ijcmas.2020.907.214 